\title{
A SCUOLA DI PARI OPPORTUNITÀ. IL SISTEMA SCOLASTICO: UN CIRCUITO DECISIVO -MA TRASCURATO- PER EDUCARE AL RISPETTO DELL'IDENTITÀ E DELLA DIFFERENZA DI GENERE ${ }^{1}$
}

\author{
Elisabetta Musi²
}

\begin{abstract}
A scuola di pari opportunità. Il sistema scolastico: un circuito decisivo -ma trascuratoper educare al rispetto dell'identità e della differenza di genere

Riassunto: La scuola può promuovere un profondo rinnovamento culturale, rivolgendosi a intere generazioni di bambini e bambine, ragazzi e ragazze e alle loro famiglie. E tuttavia, a fronte di un diffuso bisogno di superamento di modelli dominanti nell'ordine simbolico e nell'organizzazione sociale, evidenziati dalle disarmonie in famiglia, dalle tensioni che sfociano in violenze, dalle vessazioni e dalle iniquità sul lavoro, le politiche di un'istruzione attenta ai generi e di promozione di una cultura non discriminante non ricevono l'attenzione che meritano.

Nell'argomentare queste posizioni, l'intervento riepiloga per sommi capi l'orientamento delle politiche italiane e del nord Europa in relazione al binomio educazione scolastica e differenza di genere. Al contempo tenta di problematizzare alcune condizioni imprescindibili perché l'attenzione al genere possa promuovere un percorso di civiltà, di maturità e responsabilizzazione di un Paese.

Parole-chiave: Scuola, educazione, pari opportunità, discriminazioni.
\end{abstract}

An Equal Opportunities School. The School System: A Decisive Circuit -But Neglectedto Educate to Respect the Difference and Gender Identity

Abstract: School can foster a deep cultural renewal by addressing whole generations of children and teenagers, along with their families. However, despite a widespread need to overcome prevailing patterns in symbolic order and social organization, revealed by lack of harmony at home, tensions resulting in violence, harassment and iniquities in the workplace... education policies and objectives aimed at teaching about gender citizenship and fostering nondiscrimination culture do not get the deserved attention.

By sustaining these positions, the present paper summarizes the stance of Italian and northern European policies with regard to the binomial school education and gender difference. Meanwhile, the article tries to problematize some unavoidable conditions for attention on gender to foster a path of civilization, maturity and assumption of responsibilities on the part of a country.

Key words: School, education, equal opportunities, discriminations.

${ }^{1}$ Data di ricezione: $24 / 10 / 2015$.

Data di accettazione: 20/11/2015.

${ }^{2}$ Ricercatrice, Dipartimento di Pedagogia, Università Cattolica del Sacro Cuore, Milano, Italia; $\square$ elisabetta.musi@unicatt.it. 


\section{La disattenzione alle differenze produce squilibri ${ }^{3}$}

Nel dicembre 2009 è stato pubblicato dalla rete europea Eurydice uno studio dal titolo Differenze di genere nei risultati educativi, in cui si illustrano le disuguaglianze di genere in ambito scolastico e le politiche nazionali a contrasto di stereotipi e pregiudizi ${ }^{4}$.

Lo studio contiene una rassegna delle ricerche in tema di genere e istruzione e riassume i principali risultati di studi internazionali condotti sulle differenze di genere in ambito educativo.

In estrema sintesi emerge la persistenza di un forte squilibrio tra le preferenze espresse da ragazzi e ragazze riguardo alle materie di studio (in cui i maschi scelgono la matematica e le discipline scientifiche mentre le ragazze la lettura e i percorsi umanistici). Si evidenzia inoltre l'importanza della percezione degli/delle insegnanti nel sottolineare le peculiarità maschili e femminili e l'incidenza di stereotipi e precomprensioni nei rapporti con allievi e allieve: convinzioni e credenze possono rappresentare un fattore importante -per quanto non esclusivo- per indurre disparità o promuovere equità tra i generi nella scuola; in altre parole le opinioni delle/degli insegnanti, l'eventuale reiterazione di luoghi comuni e pregiudizi, l'utilizzo di sussidi discriminanti o critici risultano decisivi nelle relazioni con alunni e alunne, ne legittimano l'accesso ai saperi e di conseguenza alle professioni, con tutto ciò che questo implica in termini di possibilità di autodeterminazione, libertà di scelta e di carriera, nonché di profitto per gli uni e le altre in professioni non tradizionalmente maschili o femminili (sia detto per inciso: ancora fortemente minoritaria è la presenza delle donne in professioni prestigiose, in posizioni apicali e ben remunerate).

Un item di ricerca assunto dalla rilevazione Eurydice è la trasmissione degli stereotipi sessisti nei libri di testo adottati presso le scuole primarie. Una significativa

\footnotetext{
${ }^{3}$ I paragrafi 1 e 2 sono stati oggetto di confronto e discussione con la studentessa Giulia Casarola, che ringrazio, in occasione della sua tesi di laurea in Scienze della formazione presso l'Università Cattolica del Sacro Cuore, sede di Piacenza.

${ }^{4} \mathrm{http} / / /$ eacea.ec.europa.eu/education/eurydice/documents/thematic_reports/120it.pdf
} 
indagine statistica è stata elaborata da Irene Biemmi nel 2009 sui libri di lettura per la classe quarta di scuola primaria di dieci importanti case editrici italiane. I risultati della ricerca rivelano che nel 59,1\% delle storie si hanno protagonisti di genere maschile e nel $37 \%$ protagoniste femminili. Facendo un semplice rapporto (59,1/37) si ottiene un numero $(1,6)$ che indica come per ogni 10 protagoniste femmine siano rappresentati 16 protagonisti maschi. Nei risultati differenziati per ogni singola casa editrice emerge come in nessuno dei dieci libri esaminati si raggiunga una perfetta parità numerica tra maschi e femmine: in tutti i libri i protagonisti maschili prevalgono numericamente su quelli femminili; solo in tre casi la percentuale dei due sessi è pressoché uguale, mentre in uno i protagonisti maschili sono il doppio di quelli femminili e in tre la relazione è di 1 a 3 . Anche tra gli attori secondari delle storie prevale il genere maschile (301 personaggi maschili, 53,1\%, e 181 personaggi femminili, 31,9\%). Facendo un paragone con i protagonisti, si arriva curiosamente a un risultato quasi identico a quello precedente. Il rapporto tra personaggi maschili e femminili (301/181) è di 1,7 . Se ne conclude che la discrepanza numerica tra i personaggi è addirittura, anche se leggermente, superiore a quella rilevata tra i protagonisti $(1,6)$. Donne e bambine risultano sottorappresentate sia nel ruolo di protagoniste che di personaggi secondari della vicenda (Biemmi 2009: 88).

Biemmi analizza inoltre gli ambienti in cui sono raffigurati i protagonisti prendendo in considerazione le immagini che accompagnano i testi. Su 156 immagini di spazi chiusi, 64 hanno protagonisti maschili $(41 \%)$ e 58 protagoniste femmine $(37,2 \%)$; ma su 177 immagini di spazi aperti, 89 hanno protagonisti maschili (50,3\%) e 34 protagoniste femminili (19,2\%) (Biemmi 2009: 108-109). Per quanto riguarda invece il ruolo professionale coperto dai protagonisti, le tipologie professionali attribuite agli uomini nei testi scolastici sono ampiamente superiori a quelle attribuite alle donne (ed è verosimile che queste rappresentazioni incidano sulle aspirazioni professionali dei bambini e delle bambine che leggono quei testi). E' evidente che se la maggior parte delle professioni (e in particolare quelle maggiormente prestigiose e appetibili) sono attribuite al genere maschile, sarà improbabile che una bambina possa aspirare a farle proprie. D'altra parte se non è mai valorizzata una figura maschile impegnata nei compiti di cura (in casa o nelle professioni) difficilmente i bambini troveranno conferma di una possibilità 
realizzativa anche per loro in quegli ambiti. In generale emerge che il $70 \%$ degli uomini lavora, contro il 56\% delle donne. Più specificatamente si contano 50 diverse professioni per il genere maschile (re, cavaliere, maestro, mago, scudiero, scrittore, dottore e poeta), mentre i lavori attribuiti alle donne sono solo 15 (maestra, strega, maga, Befana, fata, indovina, scrittrice, nobile, principessa, pittrice, attrice, castellana, nutrice, bibliotecaria, casalinga). E' evidente che i testi analizzati non forniscono pari opportunità agli alunni dei due sessi di definire i propri sogni professionali: i maschi hanno un'ampia gamma di professioni da cui attingere, alcune delle quali particolarmente gratificanti e qualificate (Biemmi 2009: 91-92). Le femmine invece hanno un numero ristretto di possibilità. Questo dimostra che le asimmetrie di genere nella rappresentazione del mondo professionale non si limitano al dato quantitativo, ma sono anche, e soprattutto, di tipo qualitativo. I bambini lettori vengono incentivati a "puntare in alto", attraverso rappresentazioni che offrono loro un'ampia possibilità di scelta e modelli particolarmente gratificanti a cui ispirarsi, mentre per le bambine vale l'esatto contrario (Biemmi 2009: 94). Nel complesso, le case editrici dimostrano uno scarso impegno nell' affrontare il tema della parità tra i generi, mostrando di non equilibrare nemmeno numericamente i protagonisti dei due sessi (Biemmi 2009: 118).

Ponendo in ideale continuità questa ricerca con l'analisi compiuta dal Ministero dell'Istruzione, dell'Università e della Ricerca (Miur Ufficio di Statistica) relativa alle iscrizioni al primo anno delle scuole primarie, secondarie di primo e secondo grado del sistema educativo di istruzione e formazione italiano ${ }^{5}$, si evince che le studentesse frequentanti le scuole secondarie di secondo grado nell'anno scolastico 2014-2015, sembrano optare preferibilmente verso gli indirizzi liceali (scelti dal $68 \%$ delle studentesse contro il 32\% degli studenti) con una particolare propensione ad imparare le scienze umane e le lingue, sia moderne sia classiche. Gli studenti preferiscono dotarsi di competenze tecnologiche e conoscenze scientifiche e informatiche, e pertanto aspirano a frequentare maggiormente gli istituti tecnici per il settore tecnologico $(83,9 \%$ contro $16,1 \%$ delle studentesse). Nel caso scelgano un liceo questo è scientifico e, più

\footnotetext{
${ }^{5}$ Analisi Miur- Ufficio di Statistica, Le iscrizioni al primo anno delle scuole primarie, secondarie di primo e secondo grado del sistema educativo di istruzione e formazione, www.miur.it, aprile 2014: 5, 9.
} 
precisamente, con l'opzione "scienze applicate" e "sezione sportiva". Anche gli istituti professionali sono oggetto di un maggior numero di scelte da parte dei ragazzi, in particolare la presenza maschile è maggiore nel settore Industria e Artigianato $(75 \%$ contro il $25 \%$ delle studentesse).

$\begin{array}{llll}\text { Indirizzi di studio } & \text { Femmine } & \text { Maschi } & \text { Totale } \\ \text { Totale Licei } & \mathbf{6 8 , 0} & \mathbf{3 2 , 0} & \mathbf{1 0 0 , 0} \\ \text { Liceo Classico } & 68,0 & 32,0 & 100,0 \\ \text { Liceo linguistico } & 81,1 & 18,9 & 100,0 \\ \text { Liceo Scienze Umane } & 89,7 & 10,3 & 100,0 \\ \text { Totale Istituti Tecnici } & \mathbf{3 2 , 2} & \mathbf{6 7 , 8} & \mathbf{1 0 0 , 0} \\ \text { Istituti professionale- } & \mathbf{2 5 , 0} & \mathbf{7 5 , 0} & 100,0 \\ \text { Settore Industria } & & & \\ \text { Artigianato } & & \mathbf{5 1 , 4} & \mathbf{1 0 0 , 0} \\ \text { Totale iscritti } & \mathbf{4 8 , 6} & & \end{array}$

Il quadro complessivo indica una segregazione formativa che conduce gli alunni dei due sessi a orientarsi gli uni verso indirizzi scientifico-tecnologici e le altre verso indirizzi umanistico-letterari. Questo dato è confermato su base europea anche dalla studio Eurydice. Sebbene non esistano barriere formali all'ingresso femminile nei vari campi del sapere, le studentesse continuano a limitare i propri interessi all'interno degli ambiti considerati tradizionalmente di pertinenza femminile quali l'educazione e la cura, ambiti ai quali i ragazzi preferiscono sottrarsi. Le scelte delle ragazze e dei ragazzi sembrano dunque condizionate dal permanere di secolari stereotipi sessisti impressi dalla famiglia e confermati dalla scuola. 


\section{Un curriculum nascosto più forte di quello dichiarato}

È come se a scuola agissero simultaneamente due curricoli, in contrasto tra loro: un primo esplicito, visibile e identico per maschi e femmine che convivono in classi miste, e un secondo nascosto, tacito, inconsapevole, nel quale si collocano per ragazzi e ragazze le immagini di sé come studenti e studentesse e le attese delle famiglie sul futuro dei propri figli e figlie (Biemmi 2009: 36-39).

Sembra dunque che l'educazione al genere passi attraverso esperienze e interazioni irriflesse: gli/le insegnanti non sono immuni dall'assumere visioni precostituite della realtà, rischiando di veicolare, per lo più inconsciamente, stereotipi di genere che influenzano in modo significativo la pratica professionale e i processi di apprendimento. È necessario perciò porre l'attenzione sul curriculum nascosto, e non solo sull'imprescindibile lavoro di revisione dei curricoli scolastici e dei programmi universitari che insegnanti e ricercatrici impegnate nella didattica degli studi di genere portano avanti. La trasmissione del sapere fa leva su metamessaggi e relazioni interpersonali che inevitabilmente condizionano l'apprendimento dei contenuti e che se rimangono invisibili, ignorate o trascurate continuano a inficiare, a contrastare, smentire i messaggi espliciti, reiterando discriminazioni e disuguaglianze. A questo proposito la sociologa Graziella Priulla ha preso in considerazione i racconti narrati nella scuola dell'infanzia, dai quali bambini e bambine attingono gli archetipi millenari e le metafore della condizione umana. Gli archetipi custodiscono significati universali che trascendono la loro storicità. Le fiabe popolari tradizionali, però, riflettono anche il sistema patriarcale in cui sono state concepite, che sancisce una netta corrispondenza tra differenze di genere, ruoli, compiti e possibilità lecite o negate (Priulla 2013: 222-223).

In altre parole proprio perché il curriculum nascosto trasmette a ragazzi e ragazze un insieme di messaggi che spesso rafforzano la stereotipizzazione di genere nel processo sociale di istruzione, la domanda deve essere sia "cosa si insegna?" (cioè quali argomenti, stimoli o riflessioni sottoporre), sia "come lo si insegna?", al fine di cercare le modalità 
didattiche più adeguate per trasmettere saperi e conoscenze. Se educare al genere significa educare a una relazione trasformativa nei confronti di se stessi e del mondo, evidentemente le prassi pedagogiche tradizionali non si configurano come le più adeguate per raggiungere questo obiettivo (Gamberi, Maio, Selmi 2011: 133).

Ripensare la relazione educativa è dunque un passaggio indispensabile per progettare e realizzare attività didattiche che affrontino in modo esplicito le tematiche di genere secondo una logica multidisciplinare e di attivo coinvolgimento di alunne e alunni.

Rivedere la relazione educativa in ottica di genere significa saper adottare un approccio trasversale al tema del genere che può essere assunto in tutti i campi disciplinari e da docenti di tutti gli ordini scolastici, tanto più che l'insegnamento sensibile al genere rappresenta uno strumento importante per la gestione della classe, uno strumento che può avere forti ripercussioni sia sul rapporto fra allievi/e e insegnanti sia sul rapporto fra gli/le allievi/e stessi/e.

Articolare in modo più complesso l'interrogativo "come si insegna?" significa dedicare spazio e attenzione a:

l'autoriflessione;

il linguaggio che si usa;

la supervisione pedagogica in ottica di genere;

l'utilizzo di metodologie attive che permettano alle identità dei soggetti in formazione di esprimersi trovando le vie più consone per costruire il proprio stile di apprendimento e relazionale.

Il momento dell'autoriflessione si attua sul piano individuale e accompagna la pratica dell'insegnamento prima, durante e dopo la presenza in classe. L'autoriflessione permette di indagare gli atteggiamenti, i valori, le teorie e le supposizioni riguardo all'insegnamento e all'apprendimento che gli insegnanti si costruiscono nel tempo e che portano con sé in classe (Sapegno 2014: 16-17). Il primo passo è quindi quello di sottrarsi alla neutralità esplicitando il proprio posizionamento (Gamberi, Maio, Selmi 2011: 26). 
Liberarsi dall'illusione della neutralità significa rigettare l'idea che sia possibile cancellare il proprio genere per proporsi come modello di un apprendimento neutro. Il rischio dell'aspirazione alla neutralità è infatti quello di rendere inconsapevolmente secondari i saperi di genere, riportandoli esclusivamente a una dimensione privata dell'esistenza, continuando a trasmettere messaggi educativi che rispecchiano valori tradizionali, luoghi comuni e pensiero dominante.

Un secondo passo derivante dall'autoriflessione è riconoscere il proprio essere situati nella storia e nella cultura: diventare donna o uomo è stato nel tempo un processo di trasgressione, negoziazione o omologazione rispetto ai codici imperanti e la dimensione dell'identità di genere ha plasmato, inciso, rivoluzionato l'essere nel mondo.

Riflettere sul proprio posizionamento in termini di genere significa anche saper costruire la propria relazione con i bambini, le bambine e gli/le adolescenti esplicitando il proprio percorso di formazione e condividendo quegli aspetti della propria biografia che sono stati significativi sotto il profilo del genere, come per esempio le letture, gli incontri, i problemi, i successi ecc. Ragionare sul proprio ruolo di docenti significa avviare un'analisi interiore dei valori, degli atteggiamenti, degli stereotipi rispetto all'identità di genere di cui si può essere portatori e portatrici inconsapevoli (Sapegno 2014: 18).

Un'ulteriore caratteristica da tenere sotto controllo nella relazione educativa è il linguaggio che si utilizza. Come ricorda la filosofa Luce Irigaray, "parlare non è mai neutro" (Irigaray 1991), e porre attenzione alla differenza di genere nel linguaggio significa svelarne la natura intimamente androcentrica mettendo in risalto il rapporto che esiste tra parola, valori e costruzione della realtà. La diversità deve essere riconosciuta come luogo particolare che implica per uomini e donne modalità diverse di esperienze, percorsi non simmetrici e non riducibili gli uni agli altri (Gamberi, Maio, Selmi 2011: 134). Declinare al maschile e al femminile è ad esempio una "buona prassi" che dà visibilità linguistica anche alle donne.

Un terzo passaggio importante per rispondere all'interrogativo "come si insegna?" deriva dalla supervisione pedagogica in un'ottica di genere. È per contrastare il rischio 
di perpetrare l'educazione sessista che può rivelarsi utile intraprendere una supervisione pedagogica (Oggionni 2013), uno spazio in cui l'autoriflessione del singolo insegnante si apre al confronto altrui con l'appoggio e il monitoraggio di una figura esperta sui temi del genere.

La supervisione pedagogica è uno strumento che può rivelarsi importante per affrontare in modo collaborativo e costruttivo un percorso di pedagogia di genere nella scuola, perché ha come principale finalità quella di stimolare il personale educativo a una più profonda comprensione del campo in cui si trova a operare, nonché di incidere sulla riduzione dei nodi problematici, a livello sia organizzativo sia relazionale, mediante il potenziamento di competenze individuali e collettive.

La supervisione, promuovendo una riflessività (auto)critica e un monitoraggio periodico dei risultati ottenuti, consente ai docenti di rilevare personalmente i progressi conseguiti nell'incremento sia della qualità percepita in relazione alle proprie competenze sia dell'efficacia di ciò che si fa.

Questo aiuta a creare e a consolidare uno spazio di autoriflessione con l'ulteriore vantaggio di farne una pratica di dialogo e cooperazione tra colleghi/e.

La quarta macro area attraverso cui è possibile migliorare il modus operandi riguarda le metodologie con cui si insegnano determinati contenuti. Si tratta di un ambito decisamente operativo, che si intreccia direttamente con la didattica e con la presenza in classe (Sapegno 2014: 18-20).

Ciò che favorisce la trasmissione dei saperi sottoposti al vaglio della critica di genere sono metodologie di didattica attiva e partecipata, in grado di sollecitare un apprendimento emotivo oltre che intellettivo (Gamberi, Maio, Selmi 2011: 135). Per esempio nell'attività ludica il bambino non proietta solo le identificazioni con le figure parentali, ma anche identificazioni con personaggi immaginari. Fin dalla scuola dell'infanzia, dunque, è utile saper proporre sollecitazioni e interazioni, pratiche di ascolto e riflessività, saper incoraggiare la partecipazione di tutti e stimolare la franchezza e la diversità di opinioni (Gamberi, Maio, Selmi 2011: 135-136). Si tratta di proposte di 
lavoro che sviluppano la capacità di mettersi in gioco perché interpellano il corpo, la mente e la parola (Sapegno 2014: 20-21).

\section{Destrutturare il sapere piuttosto che compensarne le lacune}

Ammettendo di superare la sterile contrapposizione tra educazione e istruzione nella sintesi secondo cui "insegnare" è ad un tempo "educare" e "istruire" (Bertolini 1996), nei processi di formazione disciplinare degli insegnanti non disgiunta da quella didattica, dove si colloca la preparazione alla lettura critica dei saperi che non sono mai neutri ma sempre esprimono un radicamento di genere, quando non addirittura un invisibile fondamento sessista? E l'insegnante che volesse agire come ricercatore sul campo e intendesse procedere con rigore e metodo in questo lavoro di svelamento, quali strumenti dovrebbe avere a disposizione? E ancora, se il sapere legato alla storia delle culture di genere avesse un peso nei processi di comprensione tra uomini e donne, se l'incidenza di stereotipi e pregiudizi nei processi di rappresentazione e comunicazione tra i sessi contribuisse a quel fenomeno drammaticamente in crescita che è la violenza alle donne, sarebbe accettabile che l'educazione alla differenza fosse discrezionale, lasciata all'intraprendenza personale, sottratta al confronto sistematico, alla valutazione rigorosa e documentata? (perché, sia detto per inciso, non c'è un obbligo a questo tipo di formazione a nessun livello di ordine e grado scolastico, per quanto in ogni contesto una dimensione sia imprescindibile: che ci si rapporta sempre a bambini e bambine, ragazzi e ragazze, uomini e donne condizionati dal pensiero dominante della cultura in cui sono immersi).

Per sensibilizzare al rispetto verso la differenza di genere sono stati realizzati progetti, pubblicazioni, attivate collaborazioni e lavoro di rete tra istituzioni, enti, centri di ricerca. Tutto questo di certo ha modificato le percezioni, aumentato l'attenzione, affinato il senso critico di bambini e bambine, ragazzi e ragazze. Una rapida ricerca online permette di trovare una quantità sterminata di esperienze, sperimentazioni, percorsi formativi e didattici che hanno per oggetto la differenza di genere a scuola, i saperi, 
l'identità maschile e femminile. E tuttavia, come si è visto nel primo paragrafo, l'affermazione di pari opportunità di accesso ai saperi nella scuola è solo parzialmente riuscita. Perché?

Una delle ragioni è riconducibile al modo con cui è stato costruito il sapere. La cultura occidentale si è edificata sul pensiero della totalità o pensiero dell'Uno. Modello, misura e riferimento per l'organizzazione sociale è stato il soggetto maschile. In un parallelismo tra potere culturale e potere politico-sociale l'approccio maschile alla conoscenza si è configurato come dominio e possesso (Ciccone 2009; Bourdieu 2014); attraverso il quale il soggetto maschile ha strutturato l'ordine simbolico e materiale a proprio vantaggio. La critica a questo modello di pensiero, sostenuta anche dalle politiche scolastiche di parità, si è concretizzata in percorsi disciplinari tesi a mostrare "l'altra metà del cielo": portando cioè alla luce versioni nascoste e complementari della storia, della letteratura, e in generale dei saperi ufficiali, rileggendo segmenti di programmi ministeriali secondo una "prospettiva di genere" (Biemmi 2011). La storia è stata scritta dagli uomini, la donna sembra non essere mai realmente vissuta. Questo ha privato le donne del diritto al ricordo, relegandole nella desolazione del silenzio (Ulivieri, Cambi 1994; Ulivieri 1997; Ead. 1999). Non solo; quando esse hanno realizzato qualcosa di memorabile, l'hanno fatto adeguandosi a modelli maschili. Riportare alla luce la presenza delle donne nella storia e affermare un modo differente di essere nel mondo, è stato un passaggio decisivo e necessario, ma forse ancora pericolosamente interno ad una logica binaria, dicotomica e oppositiva, che attraverso un'operazione addizionale ha ampliato il modello rappresentazionale -dall'uno, all'uno più uno- senza troppo preoccuparsi di educare a destrutturare e ristrutturare, ad assumere responsabilmente la propria diversità, senza insegnare a concepire la propria identità come combinazione dinamica delle diverse appartenenze, evitando di esaltare l'appartenenza rigida ad un modello e agirla come strumento di esclusione.

Un'eccessiva enfasi nel tendere a riequilibrare le rappresentazioni maschili e femminili ha diffuso la convinzione che quello fosse l'obiettivo a cui tendere, mentre un guadagno di maggior rilevanza sarebbe stata la competenza a leggere criticamente e dinamicamente i processi con cui uomini e donne si sono inseriti e si inseriscono nella 
vita di una società, vi partecipano attivamente, ne determinano evoluzioni e cambiamenti, accedono alle opportunità che offre, cosa sono in grado di fare e di essere ${ }^{6}$, quale libertà sono in grado di agire (Sen 1997), come costruiscono e ricostruiscono la propria identità in quel gioco di censure e riconoscimenti che ricevono continue sollecitazioni dalla presenza dell'altro/a. Questo è ciò a cui tendono gli studi di genere, ai quali spesso le politiche di pari opportunità nella scuola si sono appellate ma in modo discontinuo e non sistematico; studi che non rappresentano un particolare settore disciplinare né rispondono ad una mera logica compensatoria riguardo alla cultura dominante, ma riguardano, piuttosto, un modo di porsi, un'ottica interdisciplinare da cui guardare alle scienze a partire dallo studio dell'identità di genere, della sua contestualizzazione storica, della costruzione sociale e culturale di entrambi i generi, e delle relazioni che intercorrono tra loro ${ }^{7}$. Ciò infatti su cui anzitutto si sono attestati i Gender Studies sono alcuni assunti fondamentali: l'essere umano è sempre un essere situato (prima di tutto in un corpo, poi in una storia, una cultura, un territorio); il preteso universalismo delle culture e delle regole sociali è un'astrazione, che, prescindendo dalla realtà, la mortifica. Le definizioni in cui periodicamente si incaglia la riflessione sul genere vanno intese come espressione del desiderio di semplificare la complessità dell'esistenza, non certo per darne ragione. Nella realtà i confini netti non esistono, ma esistono continuità e contiguità, sovrapposizioni, contaminazioni, ambivalenze, intrecci... sui quali operiamo segmentazioni, definizioni, in ragione delle quali più stabiliamo corrispondenze con le parole, più abbiamo la convinzione di conoscere. E tuttavia, così facendo, si è consolidata una pericolosa corrispondenza tra essere un genere e poter fare cose (Di Cori 2000: 1770; Bimbi 2012: 50-91). Tralasciando le criticità che questo collegamento ha determinato (per cui ora che la stessa categoria di genere si apre a nuove interpretazioni, sta crollando tutto il sistema che su di lei è stato costruito), l'emancipazione a cui per lo più i percorsi scolastici sulla parità hanno puntato si è limitata ad un aggiustamento del modello di base, con l'aggiunta di alcune specificazioni: "la donna può fare/essere anche..." e "l’uomo

\footnotetext{
${ }^{6} \mathrm{Si}$ fa esplicito riferimento qui all "approccio basato sulle capacità" secondo una prospettiva di genere proposto da Martha Nussbaum (cfr. Nussbaum 2001).

${ }^{7} \mathrm{Cfr}$. a titolo esemplificativo, nella molteplicità di pubblicazioni dedicate a quest'ambito di studio: Di Cori, Barazzetti 2001, Sapegno 2011, Jaunait 2008, Scott 2008, Bergqvist 1999, Griffin 2005, Davis \& Evans 2006.
} 
può essere/fare anche..." (il che ha prodotto come corollario problematizzazioni di rinforzo: "essere/fare così non significa essere meno donne/uomini" e interrogativi più articolati, del tipo: "è possibile accedere a universi tradizionalmente maschili/femminili senza snaturarsi, cioè senza scimmiottare, omologarsi all'altro/a ma conservando un'originalità identitaria, pur legittimandosi a mettere in atto modi di essere differenti di quelli indicati dalla tradizione?"). In realtà, tutte queste posizioni cadono in un errore epistemologico, facendo coincidere astrazione e universalismo, ed evidenziano la difficoltà di rapportarsi con l'alterità concreta, a cui viene di fatto negata la dignità della differenza: si tratta di una forma di discriminazione violenta contro la concretezza del reale, rimosso in nome di una normatività astratta. Una più feconda e rispettosa concettualizzazione dell'identità non disgiunta dalla differenza è quella che si discosta 0 addirittura punta a destrutturare quel paradigma identitario essenzialista che l'attuale dibattito sul Gender (o meglio il suo intenzionale travisamento strumentale ${ }^{8}$ ) mostra in tutti i suoi limiti: ciò che comunemente intendiamo per identità, e che colleghiamo a quella persistenza nel tempo garantita dal nome e cognome, non è la rappresentazione stabile e statica di un soggetto, ma un processo di continuo mutamento, una successione ininterrotta di nuove versioni di sé tra loro collegate, somiglianti ma non identiche, che non rinviano ad un "originale" precostituito, ad un "essenziale" vero e autentico (Remotti 2010). La pluralità e la specificità degli umani è la condizione della loro esistenza, e questo ben riassume l'inestricabile commistione di versioni del soggetto che costituiscono il suo essere, che compongono il sistema mobile e complesso della sua identità. La cui struttura è relazionale, cioè frutto delle trasformazioni che il soggetto attua o subisce nel corso degli incontri e delle esperienze che realizza. Ogni individuo nasce dal rapporto fisico di due soggetti differenti, per questo ha inscritta in sé la differenza; egli cambia e si evolve attraverso un continuo "differenziamento" dalla versione precedente (a cui quella successiva somiglia), si struttura nell'interazione con altri e altre in carne e ossa, quindi in un movimento di apertura e scoperta che rappresenta la sola libertà possibile:

\footnotetext{
${ }^{8}$ La questione sollevata è seria e merita la massima attenzione, a partire dal quesito di fondo: quale rapporto vogliamo coltivare con la nostra dimensione biologica, in un tempo in cui i confini di ciò che è "naturale" si sono profondamente ridefiniti? (cfr. Haraway 1995). La fatica di mantenersi in ricerca, con un'apertura al dialogo pacato e sereno tra differenti posizioni, ha determinato da parte di alcuni gruppi sociali irrigidimenti e chiusure, accuse violente e irragionevoli contrapposizioni.
} 
soggetta ai condizionamenti culturali eppure in grado di non esserne completamente succube. A questo processo, che dura tutta la vita, contribuiscono la storia, la cultura, la religione, l'educazione, i modelli, le vicende personali, l'essere situati in un tempo, uno spazio, un corpo. In ogni caso, non c'è mai un'aderenza totale e predefinita (prevedibile, prescrivibile) tra la dimensione biologica e quella umana (che è innanzitutto simbolica, relazionale, affettiva, spirituale). Ma è proprio nell'interpretazione creativa di questa eccedenza di senso che trova spazio e si realizza l'originalità di ognuno/a.

\section{Ripartire dalla scuola per contrastare le discriminazioni sessiste}

Il sistema scolastico rappresenta un reticolo istituzionale tra i più capillari e potenti nella cultura di un Paese. Uno dei codici con cui si esprime, interpreta lo spirito del tempo, ne fornisce supporti e correttivi sono le disposizioni legislative, che costituiscono il cuore delle politiche scolastiche. Ma quale contributo può fornire la scuola nel contrastare stereotipi e pregiudizi identitari se nei percorsi formativi dei suoi insegnanti e di conseguenza nell'educazione impartita a bambini e bambine, ragazzi e ragazze l'attenzione al genere non è oggetto di formazione istituzionale e programmatica di base, di apprendimento e considerazione specifica? Le politiche di pari opportunità nella scuola risultano ancora inefficaci per l'assenza di una riforma decisiva che prepari tutti gli/le insegnanti ad un uso non sessista della lingua italiana, ad una lettura critica del sapere, ad un'adeguata conoscenza dei processi di costruzione dell'identità/differenza personale in bambini e bambine, ragazzi e ragazze. Le politiche scolastiche risultano inoltre latitanti nel mettere a disposizione di tutte le scuole, di ogni ordine e grado, strumenti e sussidi didattici per educare al rispetto delle differenze; strumenti diversificati per età con cui promuovere competenze relazionali e una adeguata rappresentazione di uomini e donne nella storia e nei saperi. Non si prevedono infine efficaci dispositivi di collegamento della scuola con gli istituti di ricerca, per avviare sperimentazioni didattiche e percorsi di valutazione e documentazione della loro efficacia. 
Processi di svelamento dell'egemonia di un ordine simbolico neutro-universalemaschile hanno determinato cambiamenti minimi rispetto agli sforzi profusi. L'esiguità dei finanziamenti riservati alle ricerche, l'assenza di studi longitudinali, la carenza di interventi coordinati, sistemici e sistematici in ambito pedagogico non hanno consentito di cogliere nelle questioni di genere un processo di trasformazione attraverso il quale le persone possono costruire nuove forme di relazione e di condivisione.

Permane un silenzio complice della scuola che, tacendo su questi temi, finisce col legittimare l'esistente. A fronte di una pedagogia del saper fare che sembra aver conquistato luoghi e prassi scolastiche, è venuta meno una concezione della scuola come luogo di elaborazione di un saper essere, "luogo morale" in cui i cambiamenti sociali e culturali diventano discorso colto a supporto della crescita individuale.

Come già notava decenni fa Adrianne Rich (Rich 1991), la scuola è un luogo di donne, eppure vi è nelle stesse insegnanti una resistenza ad affrontare i temi e i compiti di una pedagogia sessuata, poiché essa costringe a metaletture impopolari e a ripercorrere la propria storia personale: non molte si sentono di fare questo percorso, forse la maggior parte lo considera poco interessante e inessenziale.

Abbiamo bisogno perciò di culture di autoriflessività, di reciprocità e relazione, di grammatiche con cui leggere intrecci e contaminazioni, che abbassino il livello di tensione e alzino quello del rispetto. Come scrive Barbara Mapelli: "Divenire donna o uomo in una stessa vita cambia molte volte direzione, assume significati differenti, differenti tonalità emotive, è un processo non lineare in cui la stessa persona, in momenti diversi, può identificarsi con i modelli sessuali proposti o ritrovare la propria identificazione nel rifiuto di stereotipi imposti, ma quel che è certo è che la tessitura del progetto di sé donna o uomo è un lavoro quotidiano, che raccoglie nelle proprie maglie ogni minuzia, ogni piccola scelta, ogni resa o conflitto, ribellione o complicità" (Mapelli 2008: 27).

Imparare a comprendere le possibilità e i limiti del proprio divenire è l'apprendimento di fondo per darsi nuove nascite: evoluzione di un progetto esistenziale 
che intreccia autonomia-intimità e dipendenza-riconoscimento dell'altro e di sé nell'altro (Mapelli 2008: 32).

\subsection{Per un'educazione critica}

L'apprendimento necessario riguardo all'identità e alla differenza, è quello di offrire a ognuno le risorse per comprendersi nella propria complessità, perché ciascuno apprenda ad accettare, a condividere intimamente le proprie fragilità, senza la necessità di fare riferimento a modelli forti o comunque preconfezionati. La storia insegna se non diventa trappola, risucchio del passato o ipoteca sul futuro. Servono quindi modalità di formazione situazionale continua, che partano dalla decostruzione critica delle forme irrigidite attraverso cui le identità di genere sono culturalmente e socialmente plasmate. Una formazione che alleni nel mettere in relazione l'immateriale -condizionamenti, retaggi del passato, pressioni e precarietà che inquinano il clima culturale generale- con la concretezza dell'esistere, la pedagogia del ciclo di vita con la pedagogia della famiglia, così da comprendere come i vissuti familiari costituiscano un calco imprescindibile nella costruzione di sé, la pedagogia sociale con la pedagogia scolastica, per comprendere come ciò che alimenta le relazioni tra soggetti siano ambienti apparentemente lontani o esterni ai contesti quotidiani -come i messaggi mediatici-, eppure direttamente incisivi. Affinché il cambiamento attecchisca nella realtà quotidiana, nei comportamenti interpersonali e in tutti gli ambiti dell'esistenza, è necessario operare con più radicalità nelle forme rappresentazionali (rete, media) -che dall'esterno rimbalzano a scuola e lì non di rado vengono ratificate-, che più di qualsiasi altre intervengono nell'immaginario inducendo pensieri e comportamenti. Non è solo la scuola responsabile del perpetuarsi di ruoli e stereotipi sessuali, ma nel momento in cui non li sottopone a critica e semplicemente li ignora, legittima quella che potrebbe essere definita l'evidenza invisibile. Attraverso la finzione (mediatica, virtuale) della neutralità si affermano norme di genere tanto più potenti quanto più restano nel non detto. Occorre operare allora una torsione di sguardo con cui la didattica non solo rilegge criticamente i contenuti disciplinari soppesandone 
tacite autorizzazioni e censure -ad esempio, come si è visto, la quantità di donne e uomini rappresentati nei libri di testo, le attività a cui sono associati...- ma sa seguire percorsi imprevedibili e complessi che si snodano tra le vicende di ogni bambino e bambina, la ricerca di forme e mezzi con cui esprimere il potenziale evolutivo originale e proprio di ognuno.

\section{Modelli di politiche di parità a confronto}

Dalla conferenza mondiale delle donne che si è tenuta a Pechino nel 1995, nonostante uno dei primi obiettivi strategici fosse proprio la riforma del sistema educativo e la verifica della sua efficacia, gli interventi di attenzione al genere nell'ambito dell'istruzione sono rimasti decisamente in secondo piano rispetto alle politiche per le pari opportunità nel mondo del lavoro e nella rappresentanza politica istituzionale. In Italia come in Europa, infatti, il focus prevalente è sull'economia, sul lavoro e sul bisogno di incentivare l'occupazione femminile nei settori per lo sviluppo economico: delle scienze e delle tecnologie. Le azioni intraprese si sono orientate all'inclusione delle donne negli organi dirigenziali, negli ambiti decisionali, finanziari e di potere esistenti, piuttosto che a una trasformazione più profonda degli stessi. L'accezione con cui sono state intese le politiche di genere nell'istruzione si è appiattita sull'interpretazione che vede il ruolo del mondo educativo funzionale alla competitività economica e le politiche di genere in campo scolastico promotrici dell'accesso delle ragazze alle professioni scientifiche e tecnologiche. Dal trattato di Lisbona ${ }^{9}$ in poi, le tematiche di genere sono entrate sì nell'agenda della Commissione per il Consiglio europeo, ma in modo discontinuo ed eterogeneo, senza che siano previste modalità di rilevazione dell'impatto e dei risultati.

La Road Map europea 2006-2010, una sorta di tabella di marcia per le pari opportunità, denunciava come il cammino per l'acquisizione dei diritti civili per le donne

\footnotetext{
${ }^{9}$ Trattato approvato nel 2007 ed entrato in vigore nel 2009, che istituisce la Costituzione Europea, rafforza il principio democratico e la tutela dei diritti fondamentali.
} 
anche nella moderna e democratica Europa fosse ancora lontano dall'essere terminato e invitava gli Stati ad attivare concretamente una serie di progetti finalizzati alla effettiva parità. Questo documento costituiva una sorta di manifesto non solo per le istituzioni, ma anche per tutte quelle agenzie educative (scuola, associazioni, comunità civile) deputate a contribuire alla costruzione di una cultura democratica e solidale, promuovendo una presenza più significativa delle donne nei processi economici e politici. In particolare la Road Map individuava due linee di lavoro per il superamento degli stereotipi di genere: una rivolta agli studenti e una agli/alle insegnanti, facendo riferimento ad azioni di formazione dei docenti e all'orientamento di ragazzi e ragazze. Nella relazione del 2008 ma soprattutto nei documenti successivi della Commissione europea sulla sensibilizzazione al genere attraverso il sistema scolastico, venivano particolarmente marcati i temi della conciliazione e del coinvolgimento degli uomini: di nuovo l'attività culturale veniva subordinata a questi obiettivi. Alla necessità di integrare politiche di genere e politiche per l'istruzione non è stato riconosciuto un peso centrale. Ciò non significa che singoli Paesi non abbiano operato in questo senso.

È stato probabilmente il crescente fenomeno di violenza alle donne a riportare all'attenzione generale l'importanza della formazione e della scuola dal momento che il Parlamento europeo ha approvato il 12 marzo 2013 una Risoluzione sull'eliminazione degli stereotipi di genere nell'Unione europea, un documento che indica azioni concrete da intraprendere nei mezzi di informazione e nella cultura, nell'istruzione e nella formazione, nel mercato del lavoro, nel processo decisionale economico e politico. Nella parte riguardante la formazione è stata affermata la rilevanza dei programmi scolastici nel perpetuare discriminazioni di genere laddove non correttamente orientati al superamento di stereotipi sessisti. Agli Stati membri è stato perciò richiesto di valutare programmi di studi e contenuti dei libri di testo nell'ottica di una riforma complessiva che conduca all'integrazione delle questioni di genere, quale tematica trasversale, in tutti i materiali didattici, sia in termini di eliminazione degli stereotipi di genere, sia in termini di maggiore visibilità del contributo e del ruolo delle donne nella storia, nella letteratura e nell'arte, già a partire dai primi livelli dell'istruzione. Gli Stati membri sono stati altresì sollecitati a predisporre specifici corsi di orientamento, nelle scuole primarie e secondarie 
e negli istituti di istruzione superiore, finalizzati a informare i giovani in merito alle conseguenze negative degli stereotipi di genere, nonché a incoraggiarli a intraprendere percorsi di studi e professionali superando visioni tradizionali che tendano a individuarli come tipicamente "maschili" o "femminili".

\subsection{L'Italia e il nord Europa}

Esperienze significative e avanzate di politica scolastica si trovano nel nord Europa, in cui la ricerca compiuta dai Gender Studies contribuisce a guidare la formazione degli/delle insegnanti e la sperimentazione didattica. In Danimarca ad esempio gli studi di genere vantano una forte e lunga tradizione, un elevato standard di qualità della ricerca in centri specifici e all'interno dell'università, in contesti disciplinari e interdisciplinari. Come in altri Paesi, la ricerca si riferisce principalmente alle scienze sociali e alle discipline umanistiche. Sono state sviluppate -con l'importante supporto dello Stato- reti e coordinamenti nazionali di centri di ricerca e associazioni che hanno dato vita a studi nazionali e internazionali, sono stati istituiti organi e strumenti di diffusioni dei risultati conseguiti, realizzati importanti archivi e database nazionali, pubblicazioni regolari e periodiche diffuse in tutto il Paese. Nonostante i finanziamenti statali non vi è stata una “istituzionalizzazione” degli studi di genere all'interno dell’Università, per cui la Danimarca non ha un programma completo di studi di genere nelle scienze sociali o in ambito umanistico né all'interno delle diverse facoltà ai diversi livelli accademici. Ugualmente il rilevante investimento sugli studi di genere ha fatto sì che crescesse un generale interesse nel Paese per questo ambito di ricerca, per quanto molti ragazzi e ragazze che si appassionano a questo tema proseguano poi i loro studi all'estero. Va aggiunto che negli ultimi tempi in Danimarca il dibattito sull'uguaglianza si è sensibilmente affievolito e con l'indebolirsi dei movimenti femministi anche la ricerca ha assunto un carattere maggiormente teoretico, mentre sono stati fatti grandi progressi in Svezia, dove l'attuale governo e cinque dei maggiori partiti politici si definiscono femministi. In Svezia infatti dal 1980 i Centres for Gender Research (inaugurati alla fine 
degli anni Settanta da un gruppo di studio formato da studiosi delle maggiori università del Paese), hanno ricevuto un importante sostegno dallo Stato, ponendosi come punto di riferimento autorevole e importante per stimolare la ricerca interdisciplinare e sul campo. Oltre a questi centri interdisciplinari, la ricerca sulle tematiche di genere si è sviluppata nella maggior parte delle università all'interno dei dipartimenti tradizionali, come parte integrante dei diversi settori disciplinari (Bencivenga 2015: 236). Questo ha determinato l'alto livello di organizzazione della ricerca sul genere e il consolidamento degli studi di genere come disciplina a sé. La ricerca di genere si è sviluppata dunque sia come disciplina autonoma sia come settore multidisciplinare ${ }^{10}$. Negli anni Novanta questo ambito di ricerca, che ha assunto il nome di "Gender Science", si è strutturato all'interno dell'offerta universitaria e oggi corsi di laurea, master e programmi di dottorato di ricerca sulle questioni del genere sono presenti in quasi tutte le università e college del Paese. Lo Stato ha continuato a sostenere gli studi di genere perché ne ha visto l'importanza nel promuovere la parità nell'istruzione superiore, così come nella società in generale. Inoltre gli/le studiosi/e di genere hanno fondato una loro associazione, l'Associazione Nazionale dei Ricercatori di genere, con adesione individuale. Questa associazione rafforza la cooperazione tra $\mathrm{i}$ ricercatori di genere e facilita l'attivazione di ricerche e azioni congiunte qualora se ne ravvisi l'opportunità. Si può concludere pertanto come sia stato della massima importanza che i consigli di ricerca e le autorità svedesi abbiano perseguito strategie a lungo termine per lo sviluppo della ricerca di genere. Va detto tuttavia che nell'ultimo piano di ricerca da parte del governo, il tema del genere è stato poco menzionato, nonostante l'unione europea chieda espressamente che la prospettiva di genere sia inclusa in tutte le ricerche. L'esperienza norvegese ha evidenziato come $\mathrm{i}$ percorsi formativi per educatori e insegnanti debbano sviluppare specifici programmi di didattica e sostenere la formazione di competenze relazionali attente al genere (Stoltz, Jacobsen, Gemzöe and M. Fahlgren 2015: 233), promuovendo la ricerca e la diffusione di conoscenze, metodologie e strumenti di insegnamento/apprendimento in grado di

\footnotetext{
${ }^{10} \mathrm{La}$ ricerca di genere ha influenzato significativamente lo sviluppo delle conoscenze in molti campi di ricerca. Per fare alcuni esempi: il canone letterario è cambiato quando è stato esaminato da una prospettiva di genere. La ricerca di genere ha anche cambiato il modo in cui viene condotta una ricerca storica e la ricerca nel campo della medicina e biologia ha beneficiato di prospettive di genere che hanno rivelato pregiudizi sessisti nella comprensione dei risultati della ricerca.
} 
prestare attenzione alle differenze di genere e a quelle personali. Anche in Norvegia il supporto dello Stato ha significativamente contribuito allo sviluppo dei Gender Studies che a loro volta hanno positivamente influenzato politiche sociali più eque ${ }^{11}$.

Complessivamente si può dire che vi sia un modello nordico di studi di genere del tipo "bottom-up", con ricerche e progetti avviate dal basso (Danimarca), mentre i modelli svedesi e norvegesi sono basati su un alto grado di istituzionalizzazione degli studi sulla parità di genere (modello “top down”).

La crisi economica globale, tuttavia, ha indotto dei tagli alla ricerca in questi ultimi anni. Anche il programma quadro europeo Horizon 2020 ha ridotto drasticamente i finanziamenti riservati alla ricerca nelle Scienze Sociali e Umanistiche (SSH) dirottandoli verso la ricerca scientifica e tecnologica. Questo lascia pensare che la prospettiva di genere susciti ancora resistenza. Probabilmente a causa del fatto che essa sfida le norme sociali e la distribuzione del potere, rispetto ad altri ambiti di studio. È dunque importante prendere in considerazione questa resistenza nei confronti della ricerca di genere, che attesta ovunque la necessità di cambiamenti sociali. Cosa c'è di così "destabilizzante" nei progetti di educazione alle differenze da scatenare una tale opposizione? Probabilmente la libertà e la rinegoziazione del potere -a vari livelli- che essa presuppone ${ }^{12}$. Una ricerca comparativa condotta in 11 Paesi europei ha dimostrato infatti che il livello di istituzionalizzazione degli studi di genere di un Paese -Centri di ricerca specifici, alte scuole e Università- è direttamente proporzionale all'incisività delle politiche di pari opportunità di quel Paese (Griffin 2005), ovvero al livello di democrazia e alla gestione critica del potere. L'analisi sull'organizzazione sessuale della società e sull'impatto delle

\footnotetext{
11 "In Norway, state feminism has been an important dimension of the national political context for the development of gender studies. The concept of state feminism was introduced by Hernes (1987) to refer to how the Scandinavian welfare states have furthered feminism "from above" through womenfriendly welfare and gender equality policies in conjunction with "feminization from below" and feminization of the welfare state professions (Holst 2005). State feminism has created a structure of possibilities for women's and gender studies to develop and be institutionalized in the academia, while gender research has importantly influenced state politics in areas such as childcare, education and politics" (Bencivenga 2015: 234).

${ }^{12}$ I rapporti di genere, in quanto densi di tensioni attorno alle forme simboliche del potere, possono esser considerati come un campo sociale totale (cfr. Moi 1991: 1017-1049), che interseca tutti gli altri campi di ricerca. È necessario quindi aumentare le ricerche gender oriented, interrogandosi sulla riproduzione delle gerarchie sociali sessuate e sulle politiche della sessualità.
} 
politiche sessuali, permette di combattere le disuguaglianze che ancora pesano prevalentemente sulle donne.

5.2. Politiche scolastiche di parità in Italia: uno scenario confuso

In Italia la fisionomia degli studi di genere è tutt'altro che ben definita.

A fronte di importanti risultati di ricerca relativi ai Gender Studies, il bilancio sul piano della trasmissione e della formazione è segnato dalla discontinuità. Da un lato si contano momenti alti di intenso coinvolgimento tra generazioni diverse (riviste e pubblicazioni di qualità, seminari, scuole estive, convegni, dottorati); dall'altro si segnala una irregolare presenza e programmazione di corsi e gruppi di ricerca in molti importanti atenei nazionali, e scarse occasioni per le donne giovani di ricoprire ruoli di primo piano $^{13}$. Una ricognizione nelle pagine $w e b$ di atenei sparsi lungo il Paese conferma il fatto che essi sono diffusi un po' ovunque, e ricevono vivo apprezzamento da studenti e studentesse, come attestano le iscrizioni e la vivacità di proposte didattiche; segno evidente che nel periodo più recente sono stati fatti grandi sforzi per costruire percorsi di studio assai seri, che poco hanno da invidiare a quelli di altri Paesi. La loro diffusione però è merito per lo più di studiose e studiosi sensibili e non di un impegno istituzionale e politico sistematico e regolarmente finanziato.

Probabilmente l'inconfessato scetticismo che accompagna gli studi di genere è da collegare alla crisi profonda dell'intero apparato politico, istituzionale e culturale che sembra congelare ogni possibilità di cambiamento. Verso la metà degli anni Novanta, il dibattito ha provato a distinguere tra assimilazione, integrazione o autonomia degli studi di genere all'interno dell'organizzazione curricolare. A distanza di anni, dopo diversi

\footnotetext{
${ }^{13}$ Cfr. P. Di Cori nella relazione presentata al convegno "On ne naît pas... on le devient". I gender studies e il caso italiano, dagli anni Settanta a oggi, tra liberazione sessuale e nuovi tabù, Université StendhalGrenoble 3, 25-26 novembre 2010, poi sintetizzata nell'articolo online di Paola Di Cori, "Sotto mentite spoglie. Gender studies in Italia" (2013).
} 
tentativi falliti di riformare la "macchina didattica", tutte e tre queste opzioni hanno continuato a rimanere questioni irrisolte.

Le politiche scolastiche di parità dovrebbero essere sostenute e promosse dal Dipartimento per le pari opportunità (di concerto con quello preposto all'istruzione e all'educazione), che tuttavia non ha fondi per promuovere ricerche di rilevanza scientifica con impatto di sistema ${ }^{14}$, in grado di imprimere un cambio di paradigma culturale nel sistema formativo scolastico, a partire dalla formazione del personale di ogni ordine e $\operatorname{grado}^{15}$.

Attualmente è fermo al Senato un Disegno di Legge (n. 1680) presentato il 18 novembre 2014 che propone 1'"Introduzione dell'educazione di genere e della prospettiva di genere nelle attività e nei materiali didattici delle scuole del sistema nazionale di istruzione e nelle università". Il Disegno punta a "integrare l'offerta formativa dei curricoli scolastici di ogni ordine e grado con l'insegnamento a carattere interdisciplinare dell'educazione di genere, a eliminare stereotipi, pregiudizi, costumi, tradizioni e altre pratiche socio-culturali fondati sulla differenziazione delle persone in base al sesso di appartenenza e a sopprimere gli ostacoli che limitano di fatto la complementarità tra i sessi nella società" (art. 1). Prevede "l'istituzione di corsi di formazione obbligatoria o che integrano i programmi di quelli esistenti, per il personale docente e scolastico" (art. 3) e, riprendendo il progetto "POLITE" (vd. infra, nota 43) dispone che le istituzioni scolastiche di ogni ordine e grado adottino libri di testo e materiali didattici corredati

\footnotetext{
${ }^{14}$ Uno dei pochi, significativi sforzi in questo senso è stato il progetto "POLITE: Pari Opportunità nei Libri di Testo", promosso nel 1996 dalla Presidenza del Consiglio dei Ministri - Dipartimento per i diritti e le pari opportunità con l'Associazione Italiana Editori, e collocato nel IV Programma d'azione comunitaria a medio termine per le pari opportunità fra le donne e gli uomini 1996-2000. Polite è un progetto europeo di autoregolamentazione per l'editoria scolastica nato con l'obiettivo di promuovere una riflessione culturale, didattica ed editoriale il cui esito fosse quello di ripensare i libri di testo in modo tale che donne e uomini, protagonisti della cultura, della storia, della politica e della scienza fossero presenti sui libri di testo senza discriminazioni di sesso. Il progetto ha prodotto un Codice di "autoregolamentazione" e due vademecum, con l'obiettivo di riqualificare i materiali didattici in vista di una maggiore attenzione all'identità di genere. (cfr. www.retepariopportunita.it e http://www.ub.es/geocrit/-xcol/163.htm; Progetto Polite. Saperi e libertà: maschile e femminile nei libri, nella scuola, nella vita. Vademecum I e II. Milano, Associazione Italiana Editori, 2000/2001). Tuttavia il Codice di Autoregolamentazione non ha mai trovato attuazione e la situazione dei libri di testo è rimasta invariata.

${ }^{15}$ Cfr.: http://www.pariopportunita.gov.it/index.php/istituzione-e-competenze
} 
dall'autodichiarazione delle case editrici impegnate a rispettare le indicazioni contenute nel codice di autoregolamentazione "Pari opportunità nei libri di testo" (Art. 5).

Il raggiungimento della parità e il superamento delle discriminazioni sessuali, nonché delle varie forme di violenza di cui le donne e le ragazze sono vittime, è in primo luogo da costruirsi attraverso un cambiamento culturale, che punti a sradicare stereotipi e rappresentazioni statiche della realtà. L'intervento educativo è l'unico strumento in grado di restituire alla rappresentazione del mondo e dei generi profondità e complessità, uguaglianza e differenza, e l'educazione, ancor più se attenta a superare stereotipi e ad usare un linguaggio rispettoso di identità e differenze, è il mezzo più potente per cambiare il mondo e per produrre una società più giusta e meno violenta.

\section{Attrezzarsi per affrontare le resistenze}

Il ruolo degli/delle insegnanti è sicuramente centrale nelle pratiche educative scolastiche che facciano proprie le questioni delle differenze di genere.

Ma è possibile educare al rispetto della differenza, alla decodifica di stereotipi e pregiudizi se gli/le insegnanti non hanno affrontato un percorso specifico nella formazione di base e continua? Se non dispongono di sussidi e strumenti didattici utili a svelare la finta neutralità dei saperi? Se non sanno rassicurare e anzi coinvolgere i genitori nell'utilità di questo investimento? È possibile avviare pratiche di formazione situazionale per sperimentare nuovi modelli più rispettosi delle differenze se la formazione di base è per lo più distante da una pedagogia critica sul genere, si propone come impersonale, assai poco sperimentale, verificata e aperta al dialogo con le diverse storie di ragazzi e ragazze, con le diverse biografie, attraverso un approccio narrativo e autobiografico? Educare al riconoscimento della differenza di genere nella sua vicenda storica e come pratica dei diritti, quali competenze richiede? E quale può essere il ruolo dell'Università in questa formazione degli/delle insegnanti? Quanto può essere decisiva l'attivazione di ricerche che raccordino i diversi saperi disciplinari, spazi e soggetti intra 
ed extrascolastici; che si incentrino sugli agenti di mutamento dentro la scuola -ad esempio riguardo alla femminilizzazione di tanta parte dei percorsi scolastici-e fuori, in relazione alle professioni, alla cooperazione in famiglia, al contrasto di iniquità e violenze?

La sensazione è che su questi temi si inizi sempre da capo. Di certo la forza di stereotipi e pregiudizi come coesivo sociale contrasta la fragilità di pensieri e pratiche nascenti, ma forse conviene prendere atto che la posta censurata è più alta di quella esplicita e che -come ha ricordato M. Grazia Contini in un libro di alcuni anni fa-dietro l'ostentato desiderio di alleanze, si cela una ben più ferrea volontà di disalleanze (Contini 2012). Da cui, forse, occorre ripartire.

\section{Riferimenti bibliografici}

Bergqvist, Christina et al. (eds.). 1999, Equal Democracies? Gender and Politics in the Nordic Countries. Oslo: Scandinavian University Press.

Bertolini, Piero. 1996. Dizionario di pedagogia e scienze dell'educazione. Bologna: Zanichelli.

Biemmi, Irene. 2009. Genere e processi formativi. Sguardi femminili e maschili sulla professione di insegnante. Pisa: Edizioni ETS.

2011. Educazione sessista. Stereotipi di genere nei libri delle elementari. Torino: Rosenberg \& Sellier.

Bimbi, Franca. 2012. “Genere. Dagli studi delle donne a un'epistemologia femminista tra dominio e libertà": AG About Gender -Rivista internazionale di studi di genere, 1: 50-91, http://www.aboutgender.unige.it/index.php/generis/article/view/11/11. [4 ottobre 2015]

Bourdieu, Pierre. 2014. Il dominio maschile. Milano: Feltrinelli.

Ciccone, Stefano. 2009. Essere maschi. Tra potere e libertà. Torino: Rosenberg \& Sellier.

Contini, Mariagrazia (ed.). 2012. Dis-alleanze nei contesti educativi. Roma: Carocci.

Davis Kathy, Mary Evans and Judith Lorber (eds.). 2006. Handbook of Gender and Women's Studies. London: Sage. 
Di Cori, Paola. 2000. "Genere e/o gender? Controversie storiche e teorie femministe". Generi di Traverso. Eds. A. Bellagamba et al. Vercelli: Mercurio.

, Donatella Barazzetti. (ed.). 2001. Gli studi delle donne in Italia. Una guida critica. Roma: Carocci.

2013. "Sotto mentite spoglie. Gender studies in Italia". Cahiers d'études italiennes [Online], 16, 2013. http://cei.revues.org/1055. [5 ottobre 2015].

Gamberi, Cristina, Maria Agnese Maio, Giulia Selmi. 2011. Educare al genere. Riflessioni e strumenti per articolare la complessità. Roma: Carocci.

Bencivenga, Rita. (ed.). 2015. "Perspectives on gender studies in Denmark, Norway and Sweden (tavola rotonda)": AG About Gender - Rivista internazionale di studi di genere. L'eteronormatività tra costruzione e riproduzione, 4: 229-251. http://www.aboutgender.unige.it/index.php/generis/article/view/290/213. [26 ottobre 2015].

Griffin, Gabrielle. 2005. “The Institutionalization of Women's Studies in Europe”. Doing Women's Studies. Employment Opportunities, Personal Impacts and Social Consequences. Ed. Gabrielle Griffin. London: Zed Books.

Haraway, Donna. 1995. Manifesto cyborg. Donne, tecnologie e biopolitiche del corpo. Milano: Feltrinelli.

Irigaray, Luce. 1991. Parlare non è mai neutro. Roma: Editori Riuniti.

Jaunait, Alexandre et al. (eds.) 2008. Introduction aux Gender Studies. Manuel des études sur le genre. Bruxelles: De Boeck. 7-10.

Mapelli, Barbara. 2008. "Adolescenti e differenze di genere. Ricerche di identità". Educare con differenza. Modelli educativi e pratiche formative. Eds. Ivana Padoan, Maria Sangiuliano.Torino: Rosenberg \& Sellier.

Moi, Toril. 1991. "Appropriating Bourdieu: Feminist Theory and Pierre Bourdieu's. Sociology of Culture", New Literary History, vol. 22, no. 4: 1017-1049.

Nussbaum, Martha C. 2001. Diventare persone. Donne e universalità dei diritti. Bologna: il Mulino.

Oggionni, Francesca. 2013. La supervisione pedagogica. Milano: FrancoAngeli.

Ojeda, Alba, Julieta y Rosa María Jiménez Catalán. 2010. "Vocabulary Gender Patterns in EFL compositions: A Cross Sectional and Longitudinal Study". Porta Linguarum, 13: 9-28.

Priulla, Graziela. 2013. C’è differenza. Identità di genere e linguaggi: storie, corpi, immagini e parole. Milano: Franco Angeli. 
Remotti, Francesco. 2010. L'ossessione identitaria. Roma-Bari: Laterza.

Rich, Adrienne. 1991. Segreti, silenzi, bugie. Milano: La Tartaruga.

Sapegno, Maria Serena. 2011. Identità e differenze. Introduzione agli studi delle donne e di genere. Roma: Mondadori.

.2014. La differenza insegna. La didattica delle discipline in una prospettiva di genere. Roma: Carocci.

Scott, Joan (ed.). 2008. Women's Studies on the Edge. Durham (NC): Duke University Press.

Sen, Amartya. 1997. La libertà individuale come impegno sociale. Roma-Bari: Laterza.

Stoltz, Pauline. et al. 2015. "Perspectives on gender studies in Denmark, Norway and Sweden ": AG About Gender - Rivista internazionale di studi di genere, 4: 229-251, http://www.aboutgender.unige.it/index.php/generis/article/view/290/213. ottobre 2015].

Ulivieri, Simonetta (ed.). 1999. Le bambine nella storia dell'educazione. Roma-Bari: Laterza. Italia. , Franco Cambi (ed.). 1994. I silenzi nell'educazione. Firenze: La Nuova 1997. Educare al femminile. Pisa: ETS. 